\title{
VITRIFICACIÓN DE EMBRIONES BOVINOS PRODUCIDOS IN VITRO
}

\section{VITRIFICATION OF BOVINE EMBRYOS PRODUCED IN VITRO}

\author{
Orlando Ramírez ${ }^{1}$, Sandra Bernal ${ }^{2}$ \\ ${ }^{1}$ DVM, M.Sc., Profesor Auxiliar Facultad de Ciencias Pecuarias. Universidad de Ciencias Aplicadas y Ambientales U.D.C.A. \\ Calle 222 No. 55-37, Bogotá, Colombia. Correo electrónico: orramirez@udca.edu.co; ${ }^{2}$ DVMZ, Esp, M.Sc., Profesor auxiliar \\ Facultad de Ciencias Pecuarias. Universidad de Ciencias Aplicadas y Ambientales U.D.C.A. Calle 222 No. 55-37, Bogotá, \\ Colombia. Correo electrónico: sabernalu@udca.edu.co
}

Rev. U.D.C.A Act. \& Div. Cient. 15(2): 419 - 429, 2012

\section{RESUMEN}

En Colombia y en el mundo la producción de embriones in vitro, a nivel comercial, se ha incrementado en los últimos años. La transferencia de los mismos, se realiza, principalmente, en fresco, debido a las posibles lesiones causadas por los procesos de criopreservación. La vitrificación es una herramienta útil en tecnologías de reproducción asistida (TRA), que se emplea en medicina humana y en veterinaria, que puede ser usada como alternativa para la criopreservación de germoplasma (oocitos, espermatozoides y embriones). Pese a los grandes avances que se han realizado en el desarrollo de dicha técnica, su aplicación es escasa en la industria de producción de embriones bovinos y se emplea, básicamente, en proyectos de investigación. En esta revisión, se discuten diferentes aspectos involucrados en el proceso de vitrificación de embriones in vitro, algunos de los resultados obtenidos, las ventajas y las principales limitantes de la misma.

Palabras clave: Criobiología, criopreservación, FIV, ganadería.

\section{SUMMARY}

Around the world and in Colombia the in vitro cattle embryo production has increased in the last years. The embryo transfer of in vitro produced embryos is mainly realized with fresh embryos, due to the possible injuries induced during the cryopreservation process. Vitrification is a tool applied in assisted reproductive technologies (ARTs) and it is used as an alternative for germplasm cryopreservation (sperm, oocytes and embryos) in human and veterinary medicine. Despite major efforts focused on the development of the technique, its commercial application is scarce in the embryo production industry and it is employed mainly in research. In this review, different aspects involved in the in vitro embryo vitrification technique, such as protocols, main advantages, limitations and some results, are discussed.

Key words: Cattle, cryopreservation, cryobiology, IVF.

\section{INTRODUCCIÓN}

La producción de embriones bovinos in vitro ha aumentado, globalmente, durante la última década; para el 2010, la IETS reportó la transferencia de 339.685 embriones bovinos, a nivel mundial (Stroud, 2011). En Suramérica, la producción de embriones es cerca del $35 \%$ de la producción global e, incluso, en algunos países, como Brasil, la producción de embriones in vitro ha superado y remplazado los procedimientos convencionales de superovulación (Pontes et al. 2010).

En Colombia, la producción de embriones obtenidos aplicando protocolos de superovulación (Bolívar \& Maldonado, 2008), así como los embriones producidos bajo condiciones in vitro (Chacón, 2001), se han intensificado desde su introducción, a finales de los años 80's y 90's, respectivamente; sin embargo, no existen datos sobre el número total de embriones y las tasas de preñez obtenidas (Bolívar \& Maldonado, 2008). Un elevado desarrollo en la producción de embriones requiere, por una parte, de una amplia disponibilidad de hembras receptoras, previamente sincronizadas y listas para ser transferidas y, por otra, mantenimiento de las receptoras, que influyen, notoriamente, en los costos de producción, por lo tanto, una alternativa para incrementar la flexibilidad de los procesos de transferencia 
de embriones es la criopreservación, evitando, de esta manera, la necesidad de su transferencia a la receptora en fresco. Tradicionalmente, para criopreservar embriones producidos in vivo, se utiliza la técnica de congelación que requiere de un equipo especializado para su realización; sin embargo, es sabido que los embriones producidos in vitro poseen una calidad inferior a los embriones producidos in vivo. Se ha identificado que los producidos in vitro pueden diferir de sus homólogos in vivo en morfología, en metabolismo, en expresión génica, en perfiles de metilación y en criotolerancia, entre otros. Dichas diferencias pueden influenciar la capacidad de desarrollo del mismo (Iwasaki et al. 1990; De la Fuente \& King, 1997, Rizos et al. 2008; Hansen et al. 2010; Stinshoff et al. 2011; Bernal et al. 2012). La congelación, como técnica de preservación, puede afectar la posterior viabilidad de los embriones producidos in vitro (Massip et al. 1995; Stinshoff et al. 2011), por lo tanto, se prefieren las transferencias de embriones in vitro en fresco a las receptoras, cuando solamente se tiene acceso a este método de criopreservación, con el ánimo de obtener tasas de preñez satisfactorias.

En estos casos, la vitrificación ha probado ser una alternativa efectiva para la criopreservación de este tipo de embriones (Wurth et al. 1994; Palasz et al. 1997; Martínez et al. 2002; Stinshoff et al. 2011). La vitrificación es un proceso físico de solidificación utilizado para conservar órganos, tejidos y embriones (Palma, 2008). Rall \& Fahy (1985) fueron los primeros en reportar la eficacia de la vitrificación en la criopreservación de embriones de ratón. Durante la vitrificación, el embrión al ser enfriado no se cristaliza sino que se produce una solidificación, pasando del estado líquido a un estado sólido no estructurado, similar al vidrio (Huang et al. 2007). La vitrificación es un procedimiento de criopreservación más simple que la congelación lenta y se ha utilizado en embriones de búfalos (Manjunatha et al. 2009), ovinos (Martínez et al. 2006), cabras (El-Gayar \& Holtz, 2001), equinos (Eldridge-Panuska et al. 2005), animales silvestres (Piltti et al. 2004) y en humanos (Elnahas et al. 2010). A pesar de ser una técnica simple, de bajo costo, con aparentemente buenos resultados, pocos trabajos se han publicado sobre este tema en el país y vagamente se conoce sobre sus beneficios, bajo nuestras condiciones (Serrano et al. 2002; Rodríguez \& Jiménez, 2011). Adicionalmente, los estudios publicados, a nivel mundial, varían en cuanto a protocolos y resultados obtenidos, por lo tanto, el objetivo del presente trabajo es proveer una revisión de las principales ventajas, variables, procedimientos y eficiencia de la vitrificación de embriones bovinos producidos in vitro.

Características de la vitrificación: Durante la vitrificación, la velocidad de enfriamiento es rápida $\left(107^{\circ} \mathrm{C} / \mathrm{seg}\right)$ y la concentración utilizada de crioprotectores es alta, pero en bajo volumen. Estas características hacen que los efectos tóxicos y las lesiones osmóticas, causadas por el crioprotector, disminuyan. Igualmente, al minimizar el volumen de la muestra, combinada con un enfriamiento acelerado, permite reducir la concentración de crioprotectores y la probabilidad de formación de cristales de hielo intracelular; es una de las crioinjurias más comunes (Cuadro 1) (Arav, 1992; Vajta et al. 1998; Hamawaki et al. 1999; Vajta, 2000; Visintin et al. 2002; Vajta \& Kuwayama, 2006; Yavin \& Arav, 2007).

Diversos protocolos de vitrificación han sido aplicados para criopreservar embriones bovinos in vitro, en los últimos años. Estos protocolos difieren en el tipo y la concentración del crioprotector utilizado, en el número de pasos de equilibrio, en las técnicas de almacenamiento y en los tiempos de

Cuadro 1. Principales ventajas de la vitrificación.

1. Contacto directo entre los embriones y el nitrógeno líquido.

2. No hay cristalización del embrión.

3. Utiliza altas concentraciones de crioprotector que disminuye el periodo de exposición del embrión a los crioprotectores.

4. Los procesos de vitrificación - desvitrificación son rápidos.

5. El uso de pequeños volúmenes provee un incremento significativo en la tasa de enfriamiento.

6. Las tasas de enfriamiento, se pueden dar entre 15,000 y $30,000^{\circ} \mathrm{C} /$ minuto.

7. Se minimizan las crioinjurias por los cambios osmóticos.

8. Se reduce el tiempo del procedimiento de criopreservación (entre 2- 10 min).

9. Los protocolos son sencillos.

10. Se eliminan los costos de adquisición de equipos.

Modificado de Liebermann et al. 2002. 
exposición del embrión, en cada una de las etapas del proceso (Park et al. 1999; Nguyen et al. 2000; Martínez et al. 2002; Dattena et al. 2004).

Tipos de crioprotectores: Los embriones para ser criopreservados necesitan ser deshidratados parcialmente para evitar la formación de cristales de hielo (cristalización) y de gradientes de concentración, los cuales, lesionan las estructuras citoplasmáticas y provocan lesiones osmóticas y tóxicas en la membrana celular (Yavin \& Arav, 2007). Esta deshidratación, se logra incorporando un agente crioprotector al medio de congelación (Celestinos \& Gatica, 2002). Los crioprotectores previenen la deshidratación total y la degeneración proteica, causada por la congelación del agua intra y extracelular, durante el proceso de congelación (Van Wagtendonk de Leeuw et al. 1997).

En la criopreservación de embriones, se pueden utilizar dos clases de crioprotectores o la combinación de estos:

- Permeables o intracelulares: Son crioprotectores de bajo peso molecular e incluyen: glicerol, dimetilsulfóxido (DMSO), etilenglicol (EG), 1-2 propanediol, etanol, propilenglicol (Martínez et al. 2006; Palma, 2008). Estos compuestos deshidratan la célula penetrándola para ayudar a proteger el citoplasma (Miyake et al. 1993). El más utilizado en los procedimientos de vitrificación es el EG, ya que tiene mayor permeabilidad y relativa baja toxicidad (Sommerfeld \& Niemmann, 1999), comparado con otros crioprotectores, como el glicerol y el propilenglicol (Palasz \& Mapletoft, 1996); no obstante, altas concentraciones de estos crioprotectores no son recomendables, puesto que pueden tener efectos tóxicos e hipertónicos. Visintin et al. (2002) mencionan que cuando se utiliza una concentración de 3,6 Molar (M) de EG, las tasas de viabilidad son similares entre los embriones vitrificados y embriones no congelados; sin embargo, cuando se aumenta a 7,2 M, la concentración de EG, se presenta embriotoxicidad.

- Impermeables o extracelulares: Son crioprotectores de alto peso molecular que extraen el agua intracelular aplicando la diferencia de presión osmótica sin penetrar al embrión y concentran las macromoléculas en el citoplasma, reduciendo así la formación de hielo intracelular (Rall, 1987; Sommerfeld \& Niemann, 1999; Visintin et al. 2002; Huang et al. 2007). Dentro de estos crioprotectores, se incluyen sucrosa, glucosa, dextrosa, trealosa, polivinil-pirrolidona (PVP) y Ficoll 70 (Kasai, 1996; Martínez et al. 2002; Huang et al. 2007), siendo el más utilizado, la sucrosa.

Con el ánimo de disminuir los efectos tóxicos (shock osmótico) específicos de los crioprotectores, durante la fase de equilibrio de la vitrificación, se puede utilizar la combinación de dos o tres crioprotectores permeables e impermeables (EG+DMSO/1,2 Propanediol/Glicerol + Sucrosa) (Vajta et al. 1997; Vajta, 2000; Martínez et al. 2002; Huang et al. 2007). Estos crioprotectores, se emplean en altas concentraciones, que permite deshidratar al embrión en forma rápida (Yavin \& Arav, 2007). Físicamente, existe una correlación entre la tasa de enfriamiento y la concentración de los crioprotectores; entre más rápida la tasa, menor será la concentración de crioprotectores requeridos y viceversa (Elnahas et al. 2010).

Procedimiento de vitrificación: Para realizar la vitrificación, se requieren básicamente cuatro pasos: 1) Colocar al embrión en una solución de equilibrio (SE); 2) Poner en contacto al embrión con la solución vitrificante (SV); 3) Empacar y sumergir el embrión en nitrógeno líquido y 4) Calentar el embrión vitrificado (desvitrificación).

Respeto al primer paso, embrión en solución de equilibrio (SE): Durante la fase inicial de equilibrio, los embriones obtenidos entran en contacto con un medio de mantenimiento antes de ponerse en contacto con la solución vitrificante. Para el periodo de equilibrio, se han reportado valores de 1,5 minutos (Vajta et al. 1999), 5 min (Martínez et al. 2002; Varago et al. 2006; Huang et al. 2007), 7 minutos (Dobrinsky et al. 1991) o 10 minutos (Kasai, 1996; Martínez et al. 2006). La solución de equilibrio (SE) consiste en un medio de mantenimiento (normalmente TCM-199), suplementado con bajas concentraciones de crioprotectores (i.e. 10\% EG+10\% DMSO) (Vajta et al. 1998). En esta fase, el embrión empieza a entrar en contacto con el(los) crioprotector(es) en múltiples pasos, incrementando, gradualmente, la concentración de crioprotector, en cada uno de ellos. Se ha reportado la exposición del embrión en un solo paso (Saha et al. 1996; Walker et al. 2006), dos pasos (Vajta et al. 1998) y tres pasos (Donnay et al. 1998).

Los resultados indican que al aumentar el número de pasos de equilibrio antes de la inmersión final en la solución de vitrificación, la viabilidad de los embriones es mayor después de la desvitrificación (Kuwayama et al. 1992; Ohboshi et al. 1997; Walker et al. 2006; Manjunatha et al. 2009). Este incremento gradual en la concentración del crioprotector permite que se disminuya el estrés osmótico y el daño celular (Kuwayama et al. 1992); sin embargo, el número de pasos en la fase de equilibrio es uno de los puntos críticos que aún impiden que la vitrificación sea utilizada a nivel comercial, ya que el tiempo en cada uno de los pasos puede retrasar el proceso, cuando se trata de vitrificar un número elevado de embriones. Walker et al. (2006) evaluaron las tasas de viabilidad de los embriones in vitro, comparando 
procesos en los que se adicionaba el crioprotector, en uno o dos pasos. Las tasas de viabilidad embrionaria con un paso fueron inferiores a las obtenidas con dos pasos $(85 \%$ vs $98 \%$, respectivamente); por lo tanto, el simplificar este proceso para hacerlo en una forma más rápida podría ser inadecuado, para la posterior viabilidad de los embriones.

Con relación al segundo paso, contacto del embrión con la solución vitrificante (SV): Después de la fase de equilibrio, el embrión entra en contacto con la solución vitrificante. En este caso, la SV contiene altas concentraciones de crioprotector (i.e. 20\% EG + 20\% DMSO) (Vajta et al. 1998), pero el tiempo de contacto del embrión con el crioprotector disminuye considerablemente, puesto que existe un límite biológico en la tolerancia de las células al crioprotector, para evitar su toxicidad y la formación de cristales de hielo (Vajta et al. 1999). El tiempo de exposición de los embriones con la SV varía entre los reportes dependiendo de los crioprotectores utilizados y el tipo de embrión a vitrificar (mórula o blastocisto).

El periodo de contacto del embrión con la solución vitrificante oscila entre 20-60 segundos (Vajta et al. 1997; Vajta et al. 1998; Martínez et al. 2002; Varago et al. 2006; Walker et al. 2006; Huang et al. 2007; Manjunatha et al. 2009). Algunos consideran que la viabilidad de los embriones es mayor cuando el tiempo de exposición es menor; sin embargo, Walker et al. (2006) consideran que tiempos cortos puede ser insuficientes para permitir que niveles adecuados del crioprotector ingresen al interior de la célula y eviten la formación de cristales de hielo y la posterior muerte celular.

Concerniente al tercer paso, empaque del embrión: Diversas técnicas han sido descritas para el almacenamiento de los embriones vitrificados. Estas técnicas han sido diseñadas para mantener el embrión en un contacto íntimo con el nitrógeno líquido, con el fin de obtener tasas altas de enfriamiento, para alcanzar el estado vítreo (Ríos et al. 2010). Estas técnicas permiten el uso de pequeños volúmenes de crioprotector, con la consecuente disminución de la embriotoxicidad (Ríos et al. 2010).

Inicialmente, se utilizó para la vitrificación la pajilla plástica convencional de $0,25 \mathrm{cc}$, la cual, utiliza volúmenes grandes de crioprotector (>20 $\mu \mathrm{L}$ ) (Palasz \& Maplefot, 1996; Lewis et al. 1999; Martínez et al. 2006; Vieira et al. 2007). Posteriormente, Vajta et al. (1998) adelgazaron y estiraron por calentamiento la pajilla plástica, hasta alcanzar un diámetro interno de 0,7$0,8 \mathrm{~mm}$ y desarrollaron la denominada Pajilla abierta estirada OPS (Open Pulled Straw). Este adelgazamiento de la pajilla permite utilizar un menor volumen de crioprotector y la tasa de enfriamiento pasa de $2500^{\circ} \mathrm{C} / \mathrm{min}$, obtenidos con las pajillas convencionales, hasta los $20.000^{\circ} \mathrm{C} / \mathrm{min}$, obtenidos con la OPS (Vajta et al. 1998). Con esta sistema, el embrión es cargado en el medio de mantenimiento (volumen $0,5 \mu \mathrm{L}$ $-1 \mu \mathrm{L}$ ) por la parte más fina de la pajilla e, inmediatamente, es sumergido en nitrógeno líquido quedando en contacto directo, durante el periodo de almacenamiento (Celestinos \& Gatica, 2002). Esta exposición directa con el nitrógeno y la posibilidad de contaminación con patógenos virales durante el almacenamiento (Bielanski et al. 2003) motivaron el desarrollo de técnicas, para evitar el contacto directo del embrión con el nitrógeno líquido (sistemas cerrados), eliminando el riesgo de contaminación. A partir de allí, se desarrollaron el cryoloop (Lane et al. 1999), cryotop (Kuwayama et al. 2005; Vajta \& Kuwayama, 2006; Lucena et al. 2006), el McGill Cryoleaf (Huang et al. 2007), la super pajilla abierta estirada (Super Open Pulled Straws; SOPS) (Yu et al. 2010), entre otros.

Al evaluar la viabilidad de los embriones utilizando sistemas abiertos (OPS) vs sistemas cerrados (SOPS), Yu et al. (2010) no encontraron diferencias significativas en las tasas de viabilidad a las 72 horas post-vitrificación en los sistemas de almacenamiento abiertos (OPS) y cerrados (CPS) $(35,2 \%$ y $34,9 \%$, respectivamente). Por el contrario, en un reciente estudio realizado por los autores (datos sin publicar), se encontró que al vitrificar blastocistos expandidos producidos bajo condiciones in vitro, en medios enriquecidos con suero fetal bovino (SFB), almacenados en OPS y en Cryotop, utilizando una solución de etilenglicol (20\%) DMSO (20\%) y sucrosa (10\%) en concentraciones decrecientes, con volúmenes de 0,2 $\mu \mathrm{l}$, no se presentó re-expansión de los blastocistos, a las 24 y 48 horas post desvitrificación.

Se han aplicado otros sistemas de empaque con diferentes materiales, como el vidrio (micropipetas de vidrio estiradas, glass pulled micropipettes GPM) (Ríos et al. 2010); nylon (Matsumoto et al. 2001) o gel (Gel-loading tips GL-Tip ${ }^{\circledR}$ ) (Tominaga \& Hamada, 2001). Estas variaciones en el material de empaque pretenden utilizar elementos que permitan una tasa de enfriamiento más rápido y, por lo tanto, utilice un menor volumen de crioprotector (Ríos et al. 2010).

Finalmente, el cuarto paso calentamiento del embrión (desvitrificación): Antes de realizar la transferencia, los crioprotectores utilizados deben ser retirados en forma lenta de los embriones, ya que, una vez en contacto con los fluidos uterinos isotónicos, se produce una sobrehidratación que provoca daños celulares irreversibles con ruptura de la membrana celular y la posterior muerte embrionaria (Visintin et al. 2002; Cabrera et al. 2006).

Para el proceso de desvitrificación, los embriones vitrificados son sumergidos en una serie de soluciones hipertónicas y rehidratantes, que contienen concentraciones decrecientes de soluciones osmóticas no permeables (ib. 0,5 M Sucrosa), para mantener el equilibrio entre la presión osmótica interna y exter- 
na (Yavin \& Arav, 2007). El tiempo de exposición del embrión con las soluciones hiperosmóticas decrecientes varía entre 3 y 5 min por paso (Martínez et al. 2006). Durante el calentamiento, la sucrosa actúa como una solución buffer para evitar la excesiva hinchazón de los embriones durante la remoción del crioprotector de las células (Vajta \& Kuwayama, 2006).

Consecuencias de la vitrificación (crioinjurias): En contraste de lo que ocurre con los embriones bovinos producidos in vivo, los embriones producidos in vitro presentan un mayor daño y una capacidad disminuida de sobrevivencia después de la criopreservación (Gómez et al. 2009); sin embargo, mediante el uso de la vitrificación las lesiones producidas, se reducen considerablemente. Los embriones cuando son vitrificados pueden sufrir daños morfológicos y funcionales, denominados crioinjurias (Zeron et al. 1999; Vajta \& Kuwayama, 2006). Dentro de las crioinjurias, se encuentran alteraciones en la permeabilidad de la membrana celular, por la formación de cristales de hielo (Leibo et al. 1996), fragmentación del ADN, daño en el huso meiótico, apoptosis celular (Park et al. 2006) y expresión génica alterada (Stinshoff et al. 2011). El grado de crioinjurias que sufre el embrión depende de ciertos factores, como el tamaño y la forma de las células, la permeabilidad de las membranas y la calidad y sensibilidad del embrión (Gómez et al. 2009).

La calidad del embrión ha estado relacionada con la resistencia del embrión a la criopreservación (Rizos et al. 2003). En algunos reportes, se ha establecido que el número de células en embriones producidos in vitro puede ser menor (De la Fuente \& King, 1997) y de menor compactación (Iwasaki et al. 1990), comparadas con los embriones producidos in vivo. El efecto del estado embrionario para sobrevivir a los procedimientos de criopreservación está relacionado con el número de células (Saha et al. 1996). Gómez et al. (2009) hallaron que, después de la vitrificación, se disminuye el número de células en la masa celular interna y este factor puede explicar por qué la viabilidad de los embriones in vitro después de la criopreservación es algunas veces escasa. Estos hallazgos están de acuerdo con lo reportado por Donnay et al. (1998), quienes mencionan que existe una alta correlación ente la tasa de viabilidad embrionaria y el promedio del número de células en blastocistos, que sobrevivieron después de la vitrificación.

Ríos et al. (2010) encontraron que el estado embrionario influye en las tasas de eclosión embrionaria después de la vitrificación. Los blastocistos expandidos son menos resistentes a la vitrificación que los blastocistos (Leibo et al. 1996; Vajta et al. 1996; Donnay et al. 1998) y si artificialmente se elimina el blastocele, las tasas de viabilidad aumentan después de la vitrificación, ya que este puede alterar el potencial de criopreservación, debido a la formación de cristales de hielo durante el enfriamiento (Vanderzwalmen et al. 2002; Hiraoka et al. 2004; Huang et al. 2007). Igualmente, se han reportado mayores tasas de expansión de blastocistos cuando los embriones son vitrificados en estado avanzado de desarrollo de blastocisto que cuando se vitrifican mórulas (Vajta et al. 1996; Tominaga \& Hamada, 2001; Campos-Chillon et al. 2009).

Las diferencias en la tolerancia a la criopreservación, se pueden explicar por las diferencias entre mórulas y blastocistos, como el tamaño celular y, posiblemente, permeabilidad alterada a los crioprotectores y la concentración de lípidos (Walker et al. 2006). Periodos largos de exposición al crioprotector son nocivos para mórulas que son más sensibles a la exposición del crioprotector, necesitando, posiblemente, tiempos de exposición más cortos (Manjunatha et al. 2009). En el caso de mórulas, la concentración de lípidos es mayor que el encontrado en blastocistos, lo cual, favorece que estas sean más susceptibles a presentar crioinjurias (Saragusty \& Arav, 2011) y ha llevado a que la mayoría de los estudios de criopreservación de embriones en bovinos, se realicen alrededor del día 7, en estado de blastocisto.

Eficiencia de la vitrificación de embriones producidos in vitro: A pesar de las ya mencionadas ventajas que ofrece la técnica existen algunas variables que pueden afectar los resultados de este proceso (Cuadro 2). Algunas de estas son también compartidas con la técnica de congelación, como la toxicidad de los crioprotectores, los cuales, pueden inducir daños embrionarios dependiendo de su concentración (Saragusty \& Arav, 2011). Las otras variables están ligadas, principalmente, a la técnica como tal, es decir, al manejo del protocolo en cada laboratorio. Como ya se mencionó son diversas las modificaciones reportadas en cada publicación (procedimiento de vitrificación), las cuales, hacen que los resultados también varíen de laboratorio a laboratorio. Estas diferencias en los resultados pueden estar relacionadas con los factores mencionados anteriormente, como el tipo y la concentración de crioprotector(es) utilizado(s), la combinación de los mismos, el sistema de empaque y de métodos aplicados para la vitrificación, la tasa de enfriamiento, el volumen utilizado para la vitrificación, la calidad y el estado de embrión, así como la habilidad del técnico (Saragusty \& Arav, 2011; Vajta, 2012). Básicamente, la eficiencia de la técnica empleada puede ser probada, como ya fue mencionado, in vitro, mediante la valoración de la viabilidad del embrión morfológicamente después de la desvitrificación (re-expansión y eclosión) o, in vivo, transfiriendo los embriones desvitrificados a receptoras y, posteriormente, evaluando las tasas de gestación y de nacimientos. Diferentes trabajos reportan tasas de supervivencia embrionaria post- desvitrificación altas, de hasta el $100 \%$ (Tabla 1); sin embargo, cuando se evaluaron las tasas de gestación después de la transferencia de embriones producidos in vitro y vitrificados, estas fueron 
Cuadro 2. Variables que pueden afectar la eficiencia de la vitrificación.

1. Toxicidad de los crioprotectores.

2. Temperatura de las soluciones de vitrificación.

3. Tiempo de exposición del embrión al crioprotector.

4. Volumen de la solución crioprotectora.

5. Método de vitrificación (tasa de enfriamiento).

6. Calidad y estadio de desarrollo de los embriones.

7. Experiencia y eficiencia del personal.

8. El contacto directo entre el nitrógeno líquido y la solución de vitrificación.

Modificado de Liebermann et al. 2002.

Tabla 1: Tasas de preñez obtenidas con procedimientos de vitrificación

\begin{tabular}{|c|c|c|c|c|c|c|}
\hline \multirow{2}{*}{ \% Preñez } & \multirow{2}{*}{$\begin{array}{l}\text { Embriones } \\
\text { transferidos* }\end{array}$} & \multicolumn{2}{|c|}{ Evaluación in vitro } & \multirow{2}{*}{$\begin{array}{l}\text { Solución de } \\
\text { vitrificación }\end{array}$} & \multirow{2}{*}{$\begin{array}{l}\text { Sistema } \\
\text { empaque }\end{array}$} & \multirow{2}{*}{ Fuente } \\
\hline & & Re-expansión & Eclosión & & & \\
\hline $0 \% *$ & 6 & $18,80 \%$ & -- & $\begin{array}{l}40 \% E G+0,3 M \\
\text { SUC }\end{array}$ & $\begin{array}{l}\text { Pajilla francesa } \\
0,25 \mathrm{~mL}\end{array}$ & $\begin{array}{l}\text { Palasz et al. } \\
1997\end{array}$ \\
\hline $4,50 \%$ & 22 & $67 \%$ & $53 \%$ & $\begin{array}{l}25 \% \text { GLI }+25 \% \\
E G\end{array}$ & $\begin{array}{l}\text { Pajilla francesa } \\
0,25 \mathrm{~mL}\end{array}$ & $\begin{array}{l}\text { Donnay et al. } \\
1998\end{array}$ \\
\hline $64 \%$ & 22 & -- & -- & $\begin{array}{l}20 \% \text { EG }+20 \% \\
\text { DMSO }\end{array}$ & $\begin{array}{l}\text { Open Pulled } \\
\text { Straws }\end{array}$ & $\begin{array}{l}\text { Lewis et al. } \\
1999\end{array}$ \\
\hline $50 \%$ & 40 & $71,60 \%$ & $66,60 \%$ & $\begin{array}{l}25 \% \text { EG }+25 \% \text { GLI } \\
+0,1 \text { M SUC }\end{array}$ & $\begin{array}{l}\text { Pajilla francesa } \\
0,25 \mathrm{~mL}\end{array}$ & $\begin{array}{l}\text { Martínez et al. } \\
2002\end{array}$ \\
\hline $30 \%$ & 10 & $60 \%$ & $54 \%$ & 6,5M GLI & $\begin{array}{l}\text { Pajilla francesa } \\
0,25 \mathrm{~mL} \text { al }\end{array}$ & $\begin{array}{l}\text { Nedambale et } \\
\text { al. } 2004\end{array}$ \\
\hline $41,9 \%$ & 481 & $91,8 \%$ & $80,6 \%$ & $\begin{array}{l}35 \% \text { EG }+5 \% \text { PVP } \\
+0,4 \text { M TRE }\end{array}$ & $\begin{array}{l}\text { Microgota } \\
\text { sobre base } \\
\text { metálica }\end{array}$ & Xu et al. 2006 \\
\hline $19 \%$ & 21 & $94,80 \%$ & $75,80 \%$ & $\begin{array}{l}20 \% \text { EG }+20 \% \\
\text { DMSO }\end{array}$ & $\begin{array}{l}\text { Micropipeta de } \\
\text { vidrio }\end{array}$ & $\begin{array}{l}\text { Vieira et al. } \\
2007\end{array}$ \\
\hline $40 \%$ & 30 & $100 \%$ & $95,50 \%$ & $\begin{array}{l}16,5 \% \text { EG }+16,5 \\
\text { DMSO +0,5M SUC }\end{array}$ & Criotop & $\begin{array}{l}\text { Inaba et al. } \\
2011\end{array}$ \\
\hline
\end{tabular}

*Blastocistos

muy variables. En la tabla 1, se pueden observar resultados solución de vitrificación conteniendo $20 \%$ etilenglicol y $20 \%$ de diferentes grupos de investigación que oscilan entre 0-64\% dimetil sulfóxido (Lewis et al. 1999). Este estudio fue uno de usando diferentes sistemas de empaque y diversas soluciones los primeros estudios en reportar esta técnica con éxito en en el medio de vitrificación. Según estos resultados, las bovinos, en donde dos embriones fueron transferidos en la mejores tasas de gestación se presentaron cuando se misma receptora. Los demás valores presentados en la tabla utilizaron OPS como dispositivo de almacenamiento y una 1, no evidencian una mejoría en las tasas de gestación, lo que 
señala que pese a los grandes avances en investigación, las modificaciones realizadas al proceso no han incrementado la eficiencia del mismo. Desafortunadamente, muchos trabajos publicados no realizaron transferencia de los embriones, por lo cual, es difícil conocer si los procesos son realmente viables, es decir, que no afectan la capacidad de desarrollo del embrión, aun cuando las tasas de re-expansión y eclosión sean satisfactorias. Adicionalmente, en la mayoría de estudios que reportan transferencias, se realizaron usando grupos pequeños de embriones y receptoras, lo cual, también es cuestionable. Un estudio realizado por Xu et al. (2006) es un ejemplo importante, con un número representativo de embriones transferidos y tasas altas de viabilidad pos desvitrificación y satisfactorias de gestación (Tabla 1). En dicho estudio no se utilizó OPS sino que la vitrificación se realizó en micro-gotas sobre una superficie metálica enfriada previamente y las tasas de gestación superaron el $40 \%$. Desafortunadamente, tanto la OPS como las micro - gotas no permiten la transferencia directa del embrión, mientras que el uso de pajillas de $0,25 \mathrm{~mL}$ podría facilitar la misma, así como permitir el almacenamiento en nitrógeno líquido. En general, los protocolos de vitrificación incluyen una combinación de crioprotectores, con el ánimo de reducir la toxicidad; no obstante, el etilenglicol es el más utilizado por su alta de penetración celular estando presente en casi todos los estudios de vitrificación y evidenciando que la toxicidad, a largo tiempo y a bajas temperaturas, no supera a la congelación tradicional (Saragusty \& Arav, 2011; Vajta, 2012). A nivel sanitario, la vitrificación aun es fuente de discusión, ya que los procesos con mejores resultados, como por ejemplo las OPS y otros sistemas abiertos, aparentemente, no ofrecen seguridad en cuanto a la posible transmisión de enfermedades, por el contacto directo con el nitrógeno líquido, lo cual, también limita la aplicación comercial de la técnica y, por el contrario, los sistemas completamente cerrados aun no cuentan con la misma eficiencia de los métodos abiertos (Vajta, 2012). Estas diferencias en los procesos y puntos de vista de cada grupo de investigación hacen que la estandarización del protocolo de vitrificación sea compleja, puesto que no permiten la evolución de los protocolos más sólidos. Pese a las diferencias, se puede observar que mediante el uso de esta técnica es posible obtener tasas satisfactorias de gestación empleando embriones producidos in vitro y, posteriormente, crioconservados, aun cuando no exista un protocolo universal y aprobado para realizar la vitrificación.

\section{CONCLUSIONES Y PERSPECTIVAS}

La vitrificación es una alternativa para la criopreservación de embriones bovinos producidos in vitro. Es una técnica sencilla, que no requiere la compra de equipos de criopreservación costosos y al no formar cristales de hielo en el interior del embrión, menores lesiones se presentan durante el proceso, lo que resulta en la obtención de tasas de preñez aceptables después de la transferencia; sin embargo, en la literatura se reportan múltiples protocolos y resultados variables. Esta variabilidad en los resultados podría ser explicada por los diversos factores que pueden influir en el proceso, incluyendo el tipo de crioprotector y sus diferentes concentraciones en las soluciones de vitrificación y de desvitrificación, la curva de descenso de temperatura, el protocolo de vitrificación, el sistema de almacenamiento o la adición de suplementos durante la producción de embriones, así como la experticia del personal que la realiza.

La variabilidad en los procesos de vitrificación/desvitrificación es el reflejo de la diversidad en los protocolos de producción y de conservación de embriones en cada laboratorio, lo que, a su vez, modifica los resultados y la calidad de los embriones que se producen. Todos los factores mencionados anteriormente demuestran la falta de estandarización en los procedimientos. Por lo tanto, el reto consiste en desarrollar un procedimiento de vitrificación universal, rápido, eficiente, seguro a nivel sanitario y toxicológico, que mantenga los bajos costos, ya que en la medida en que se implementa la estandarización de los protocolos se incrementan los costos, siendo necesario el desarrollo e implementación de equipos. Cuando sea factible dicha estandarización, seguramente, la expansión de la vitrificación de embriones bovinos producidos in vitro será posible, haciéndose rutinaria la técnica a nivel comercial. Por consiguiente, se requiere mayor investigación y un consenso en la comunidad científica, para lograr tan importante avance.

Conflicto de intereses: El manuscrito fue preparado y revisado por los autores, quienes declaran que no existe ningún conflicto de interés que ponga en riesgo la validez de la información presentada.

\section{BIBLIOGRAFÍA}

1. ARAV, A. 1992. Vitrification of oocytes and embryos. En: Lauria AGF, editor. New trends in embryo transfer. Cambridge (UK). Portland Press; 255-264.

2. BERNAL, S.M.; HEINZMANN, J.; HERRMANN, D.; LUCAS-HAHN, A.; TIMMERMANN, B.; NIEMANN, H. 2012. Effects of different in vitro maturation systems on bovine embryo development. Repr., Fert. Developm. 23(1):210(Abstract).

3. BIELANSKI, A.; BERGERON, H.; LAU, P.C.; DEVENISH, J. 2003. Microbial contamination of embryos and semen during long term banking in liquid nitrogen. Cryobiology. 46(2):146-152.

4. BOLÍVAR, P.; MALDONADO, E.J. 2008. Análisis de 
costos de esquemas de transferencia de embriones bovinos utilizados en Colombia. Rev. Col. Cie. Pec. 21(3):351-364.

5. CABRERA, P.; FERNÁNDEZ, A.; BASTIDAS, P.; MOLINA, M.; BETHENCOURT, A.; DÍAZ, T. 2006. Vitrificación: Una Alternativa para la Criopreservación de Embriones. Rev. Fac. Cie. Vet. 47(1):9-23.

6. CAMPOS-CHILLON, L.F.; BARCELO, T.K.F.; SEIDEL, J.R.G.; CARNEVALE, E. 2009. Vitrification of earlystage bovine and equine embryos. Theriogenology. 71(2):349-354.

7. CELESTINOS, M.; GATICA, R. 2002. Vitrificación como técnica de crioconservación de embriones bovinos. Arch. Med. Vet. 34(2):157-165.

8. CHACÓN, J.L. 2001. Nacen primeros terneros in vitro en Colombia. Agr. Américas. 302:37-38.

9. DATTENA, M.; ACCARDO, C.; PILICHI, S.; ISACHENKO, V.; MARA, L.; CHESSA, B.; CAPPAI, P. 2004. Comparison of different vitrification protocols on viability after transfer of ovine blastocysts in vitro produced and in vivo derived. Theriogenology. 62(34):481-493.

10. DE LA FUENTE, R.; KING, W.A. 1997. Use of a chemically defined system for the direct comparison of inner cell mass and trophectoderm distribution in murine, porcine and bovine embryos. Zygote. 5(4):309-320.

11. DOBRINSKY, J.R.; HESS, F.F.; DUBY, R.T.; JROBL, J.R. 1991. Cryopreservation of bovine embryos by vitrification. Theriogenology. 37:202(Abstract).

12. DONNAY, I.; AUQUIER, P.H.; KAIDI, S.; CAROLAN, C.; LONERGAN, P.; MERMILLOD, P.; MASSIP, A. 1998. Vitrification of in vitro produced bovine blastocysts: methodological studies and developmental capacity. An. Repr. Sci. 52(2):93-104.

13. ELDRIDGE-PANUSKA, W.D.; DI BRIENZA, V.C.; SEIDEL, J.R.; SQUIRES, E.; CARNEVALE, E.M. 2005. Establishment of pregnancies after serial dilution or direct transfer by vitrified equine embryos. Theriogenology. 63(5):1308-1319.

14. EL-GAYAR, M.; HOLTZ, W. 2001. Technical note: vitrification of goat embryos by the open pulled straw method. J. An. Sci. 79(9):2436-2438.
15. ELNAHAS, A.; ALCOLAK, E.; ABU MARAR, E.; ELNAHAS, T.; ELNAHAS, K.; PALAPELAS, V.; DIEDRICH, K.; AL-HASANI, S. 2010. Vitrification of human oocytes and different development stages of embryos: An overview. Middle East Fert. Soc. J. 15(1):2-9.

16. GÓMEZ, E.; MUÑOZ, M.; RODRÍGUEZ, A.; CAAMANO, J.N.; FACAL, N.; DIEZ, C. 2009. Vitrification of Bovine Blastocysts Produced In vitro Inflicts Selective Damage to the Inner Cell Mass. Repr. Dom. An. 44(2):194-199.

17. HANSEN, P.; BLOCK, J.; LOUREIRO, B.; BONILLA, L.; HENDRICKS, K. 2010. Effects of gamete source and culture conditions on the competence of in vitro produced embryos for post-transfer survival in cattle. Repr., Fert. Developm. 22(1):59-66.

18. HIRAOKA, K.; HIRAOKA, K.; KINUTANI, M.; KINUTANI, K. 2004. Case report: successful pregnancy after vitrification of human blastocyst that had completely escaped from the zona pellucida on day 6. Hum. Repr. 19(4):988-990.

19. HUANG, J.; CHUNG, J.; LIN TAN, S.; CHIAN, R. 2007. High survival and hatching rates following vitrification of embryos at blastocyst stage: a bovine model study. Repr. BioMed. 14(4):464-470.

20. HAMAWAKI, A.; KUWAYAMA, M.; HAMANO, S. 1999. Minimum volume cooling method for bovine blastocyst vitrification. Theriogenology. 51(1):165 (Abstract).

21. INABA, Y.; AIKAWA, Y.; HIRAI, T.; HASHIYADA, Y.; YAMANOUCHI, T.; MISUMI, K.; OHTAKE, M.; SOMFAI, T.; KOBAYASHI, S.; SAITO, N.; MATOBA, S.; KONISHI, K.; IMAI, K. 2011. In straw cryoprotectant dilution for bovine embryos vitrified using cryotop. J. Repr. Developm. 57(4):437-443.

22. IWASAKI, S.; YOSHIBA, N.; USHIJIMA, H.; WATANABE, S.; NAKAHARA, T. 1990. Morphology and proportion of inner cell mass of bovine blastocysts fertilized in vitro and in vivo. J. Repr. Fert. 90(1):279-284.

23. KASAI, M. 1996. Simple and efficient methods for vitrification of mammalian embryos. An. Repr. Sci. 42(1-4):67-75.

24. KUWAYAMA, M.; HAMANO, S.; NAGAI, T. 1992. 
Vitrification of bovine blastocysts obtained by in vitro culture of oocytes matured in vitro. J. Repr. Fert. 96(1):187-193.

25. KUWAYAMA, M.; VAJTA, G.; LEDA, S.; KATO, O. 2005. Comparison of open and closed methods for vitrification of human embryos and the elimination of potential contamination Repr. Biomed. 11(5):608614.

26. LANE, M.; SCHOOLCRAFT, W.B.; GARDNER, D.K. 1999. Vitrification of mouse and human blastocysts using a novel cryoloop container-less technique. Fert. Ster. 72(6):1073-1078.

27. LEIBO, S.P.; MARTINO, A.; KOBAYASHI, J.; POLLAR, J.W. 1996. Stage-dependent sensitivity of oocytes and embryos to low temperatures. An. Repr. Sci. 42(1):45-53.

28. LEWIS, I.M.; LANE, M.W.; VAJTA, G. 1999. Pregnancy rates following transfer of in vitro produced bovine embryos vitrified by the open pulled straw (OPS) method. Theriogenology. 51(1):168 (Abstract).

29. LIEBERMANN, J.; NAWROTH, F.; ISACHENKO, V.; ISACHENKO, E.; RAHIMI, G.; TUCKER, M.J. 2002. Potential Importance of vitrification in reproductive medicine. Biol. Repr. 67(6):1671-1680.

30. LUCENA, E.; BERNAL, D.P.; LUCENA, C.; ROJAS, A.; MORAN, A.; LUCENA, A. 2006. A successful ongoing pregnancies after vitrification of oocytes. Fert. Ster. 85(1):108-111.

31. MANJUINATHA, B.; GUPTA, P.; RAVINDRA, J.; DEVARAJA, M.; NANDI, S. 2009. Effect of vitrification medium composition and exposure time on postthaw development of buffalo embryos produced in vitro. The Vet. J. 179(2):287-291.

32. MASSIP, A.; MERMILLOD, P.; DINNYES, A. 1995. Morphology and biochemistry if in-vitroproduced bovine embryos: implications for their cryopreservation. Hum. Repr. 10(11):3004-3011.

33. MARTÍNEZ, A.G.; VALCÁRCEL, A.; DE LAS HERAS, M.A.; DE MATOS, D.G.; FURNUS, C.; BROGLIATTI, G. 2002. Vitrification of in vitro produced bovine embryos: in vitro and in vivo evaluations. An. Repr. Sci. 73(1):11-21.

34. MARTÍNEZ, A.G.; VALCARCEL, A.; FURNUS, C.C.;
DE MATOS, D.G.; IORIO, G.; DE LAS HERAS, M.A. 2006. Cryopreservation of in vitro-produced ovine embryos. Small Rum. Res. 63(3):288-296.

35. MATSUMOTO, H.; JIANG, J.Y.; TANAKA, T.; SASADA, H.; SATO E. 2001. Vitrification of large quantities of immature bovine oocytes using nylon mesh. Cryobiology. 42(2):139-144.

36. MIYAKE, T.; KASAI, M.; ZHU, S.E.; SAKURAI, T.; MACHIDA, T. 1993. Vitrification of mouse oocytes and embryos at various stages of development in an ethylene glycol-based solution by a simple method. Theriogenology. 40(1):121-134.

37. NEDAMBALE, T.; DINNYE'S, A.; GROEN, W.; DOBRINSKY, J.; TIAN, X.; YANG, X. 2004. Comparison on in vitro fertilized bovine embryos cultured in KSOM or SOF and cryopreserved by slow freezing or vitrification. Theriogenology. 62(3-4):437449.

38. NGUYEN, B.X.; SOTOMARU, Y.; TANI, T.; KATO, Y.; TSUNODA, Y. 2000. Efficient cryopreservation of bovine blastocysts derived from nuclear transfer with somatic cells using partial dehydration and vitrification. Theriogenology. 53(7):1439-1448.

39. OHBOSHI, S.; FUJIHARA, N.; YOSHIDA, T.; TOMOGANE, H. 1997. Usefulness of polyethylene glycol for cryopreservation by vitrification of in vitro derived bovine blastocysts. An. Repr. Sci. 48(1):2736.

40. PALASZ, A.T.; MAPLETOFT, R.J. 1996. Cryopreservation of mammalian embryos and oocytes: recent advances. Biotech. Adv. 14(2):127-149.

41. PALASZ, A.T.; GUSTAFSSON, H.; RODRIGUEZMARTINEZ, H.; GUSTA, L.; LARSSON, B.; MAPLETOFT, R.J. 1997. Vitrification of bovine ivf blastocysts in an ethylene glycousucrose solution and heat-stable plant-extracted proteins. Theriogenology. 47(4):865-879.

42. PALMA, G. 2008. Biotecnología de la Reproducción. $2^{\mathrm{a}}$ ed. Ed Reprobiotec, Buenos Aires, Argentina. 669p.

43. PARK, S.P.; KIM, E.Y.; KIM, D.I.; PARK, N.H.; WON, Y.S.; YOON, S.H.; CHUNG, K.S.; LIM, J.H. 1999. Simple, efficient and successful vitrification of bovine blastocysts using electron microscope grids. Hum. Repr. 14(11):2838-2843. 
44. PARK, S.Y.; KIM, E.Y.; CUI, X.S.; TAE, J.C.; LEE, W.D.; KIM, N.H.; PARK, S.P.; LIM, J.H. 2006. Increase in DNA fragmentation and apoptosis-related gene expression in frozen-thawed bovine blaslocysts. Zygote. 14(2):125-131.

45. PILTTI, K.; LINDEBERG, H.; AALTO, J.; KORHONEN, H. 2004. Live cubs born after transfer of OPS vitrifiedwarmed embryos in the farmed European polecat (Mustela putorius). Theriogenology; 61(5):811-820.

46. PONTES, J.H.; SILVA, K.; BASSO, A.; RIGO, A.; FERREIRA, C.; SANTOS, G.; SANCHES, B.; PORCIONATO, J.; VIEIRA, P.; FAIFER, F.; STERZA, F.; SCHENK J.; SENEDA, M. 2010. Large-scale in vitro embryo production and pregnancy rates from Bos taurus, Bos indicus, and indicus-taurus dairy cows using sexed sperm Theriogenology. 74(8):1349-1355.

47. RALL, W.F.; FAHY, G.M. 1985. Ice free cryopreservation of mouse embryos at $-196^{\circ} \mathrm{C}$ by vitrification. Nature. 313:573575.

48. RALL, W.F. 1987. Factors affecting the survival of mouse embryos cryopreserved by vitrification. Cryobiology. 24(5):387-402.

49. RÍOS, G.L.; MUCCI, N.C.; KAISER, G.G.; ALBERIO, R.H. 2010. Effect of container, vitrification volume and warming solution on cryosurvival of in vitroproduced bovine embryos. An. Repr. Sci. 118(1):1924.

50. RIZOS, D.; GUTIERREZ-ADAN, A.; PEREZ-GARNELO, S.; DE LA FUENTE, J.; BOLAND, M.P.; LONERGAN, P. 2003. Bovine embryo culture in the presence or absence of serum: implications for blastocyst development, cryotolerance, and messenger RNA expression. Biol. Repr. 68(1):236-243.

51. RIZOS, D.; CLEMENTE, M.; BERMEJO-ALVAREZ, P.; DE LA FUENTE, J.; LONERGAN, P.; GUTIERREZADAN, A. 2008. Consequences of In vitro culture conditions on embryo development and quality. Repr. Dom. An. 43(4):44-50.

52. RODRÍGUEZ, P.; JIMÉNEZ, C. 2011. Criopreservación de embriones bovinos producidos in vitro. Rev. Fac. Med. Vet. Zoot. 58(2):107-119.

53. SAHA, S.; OTOI, T.; TAKAGI, M.; BOEDIONO, A.; SUMANTRI, C.; SUZUKI, T. 1996. Normal calves obtained after direct transfer of vitrified bovine embryos using ethylene glycol, trehalose and polyvinylpyrrolidone. Cryobiology. 33(3):291-299.

54. SARAGUSTY, J.; ARAV, A. 2011. Current progress in oocyte and embryo cryopreservation by slow freezing and vitrification. Repr. 141(1):1-19.

55. SERRANO, C.; SIERRA, R.; SÁNCHEZ, J.; RESTREPO, L. OLIVERA, M. 2002. Evaluación de dos métodos de criopreservación sobre la calidad de embriones producidos in vitro. Rev. Col. Cie. Pec. 15(3):286292.

56. SOMMERFELD, V.; NIEMANN, H. 1999. Cryopreservation of bovine in-vitro produced embryos using ethylene glycol in controlled freezing or vitrification. Cryobiology. 38(2):95-105.

57. STINSHOFF, H.; WILKENING, S.; HANSTEDT, A.; BRÜNING, K.; WRENZYCKI, C. 2011. Cryopreservation affects the quality of in vitro produced bovine embryos at the molecular level. Theriogenology. 76(8):1433-1441.

58. STROUD, B. 2011. Statistics and data retrieval Committee report. The year 2010 worldwide statistics of embryo transfer in domestic farm animals. Disponible desde Internet en: http://www.iets.org/ pdf/December2011.pdf (con acceso 01/07/2012).

59. TOMINAGA, K.; HAMADA, Y. 2001. Gel-loading tips as a container for vitrification of in vitro-produced bovine embryos. J. Repr. Developm. 47(5):267-273.

60. VAJTA, G.; HOLM, P.; GREVE, T.; CALLESEN, H. 1996. Factors affecting survival rates of in vitro produced bovine embryos after vitrification and direct in-straw rehydration. An. Repr. Sci. 45(3):191-200.

61. VAJTA, G.; HOLM, P.; GREVE, T.; CALLESEN, H. 1997. Comparison of two manipulation methods to produce in vitro fertilized, biopsied and vitrified bovine embryos. Theriogenology. 47(2):501-509.

62. VAJTA, G.; HOLM, P.; KUWAYAMA, M.; BOOTH, P.J.; JACOBSEN, H.; GREVE, T.; CALLESEN, H. 1998. Open pulled straw (OPS) vitrification: a new way to reduce cryoinjuries of bovine ova and embryos. Mol. Repr. Developm. 51(1):53-58.

63. VAJTA, G.; RINDOM, N.; PEURA, T.; HELM, P.; GREVE, T.; CALLESEN, H. 1999. The effect of media, serum 
and temperature on in vitro survival of bovine blastocyst after open pulled straw (OPS) vitrification. Theriogenology. 52(5):939-949.

64. VAJTA G. 2000. Vitrification of the oocytes and embryos of domestic animals. An. Repr. Sci. 60-61:357-364.

65. VAJTA, G.; KUWAYAMA, M. 2006. Improving cryopreservation systems. Theriogenology. 65(1):236-244.

66. VAJTA, G. 2012. Vitrification in human and domestic animal embryology: work in progress. Repr., Fert. Developm. Disponible desde Internet en: http:// dx.doi.org/10.1071/RD12118 (con acceso el 01/07/2012).

67. VARAGO, F.; SALIBA, W.; ALVIM, M.; VASCONCELOS, A.; OLIVEIRA, C.; STAHLBERG, R.; LAGARES, M. 2006. Vitrification of in vitro produced Zebu embryos. An. Repr. 3:353-358.

68. VANDERZWALMEN, P.; BERTIN, G.; DEBAUCHE, C.; STANDAERT, V.; VAN ROOSENDAAL, E.; VANDERVORST, M.; BOLLEN, N.; ZECH, H.; MUKAIDA, T.; TAKAHASHI, K.; SCHOYSMAN. R.; 2002. Births after vitrification at morula and blastocyst stages: effect of artificial reduction of the blastocoelic cavity before vitrification. Hum. Repr. 17:744-751.

69. VAN WAGTENDONK DE-LEEUW, A.M.; DEN-DAAS, J.H.G.; RAIL, W.F. 1997. Field trial to compare pregnancy rates of bovine embryo cryopreservation methods: vitrification and one-step dilution versus slow freezing and three-step dilution. Theriogenology. 48(7):1071-1084.

70. VIEIRA, A.D.; FORELL, F.; FELTRIN, C.; RODRIGUES, J.L. 2007. In-straw cryoprotectant dilution of IVP bovine blastocysts vitrified in hand-pulled glass micropipettes. An. Repr. Sci. 99(3-4):377-383.
71. VISINTIN, J.A.; MARTINS, J.P.F.; BEVILACQUA, E.M.; MELLO, M.R.; NICACIO, A.C.; ASSUMPCAO, M.A. 2002. Cryopreservation of Bos taurus vs Bos indicus embryos: are they really different? Theriogenology. 57(1):345-359.

72. WALKER, D.J.; CAMPOS-CHILLON, L.F.; SEIDEL, G.E. 2006. Vitrification of in vitro-produced bovine embryos by addition of ethylene glycol in one-step. Repr. Dom. An. 41(5):467-471.

73. WURTH, Y.A.; REINDERS, J.M.C.; RALL, W.F.; KRUIP, T.A.M. 1994. Developmental potential of in vitro produced bovine embryos following cryopreservation and single-embryo transfer. Theriogenology. 42(8):1275-1284.

74. XU, J.; GUO, Z.; SU, L.; NEDAMBALE, T.L.; ZHANG, J.; SCHENK, J.; MORENO, J.F.; DINNYÉS, A.; JI, W.; TIAN, X.C.; YANG, X.; DU, F. 2006. Developmental potential of vitrified Holstein cattle embryos fertilized In vitro with sex-sorted sperm. J. Dairy Sci. 89(7):2510-2518.

75. YAVIN, S.; ARAV, A. 2007. Measurement of essential physical properties of vitrification solutions. Theriogenology. 67(1):81-89.

76. YU, X.L.; DENG, W.; LIU, F.J.; LI, Y.H.; LI, X.X.; ZHANG, Y.L.; ZAN, L.S. 2010. Closed pulled straw vitrification of in vitro-produced and in vivo-produced bovine embryos. Theriogenology. 73(4):474-479.

77. ZERON, Y.; PEARL, M.; BOROCHOV, A.; ARAV, A. 1999. Kinetic and temporal factors influence chilling injury to germinal vesicle and mature bovine oocytes. Cryobiology. 38(1):35-42.

Recibido: Marzo 9 de 2012

Aceptado: Octubre 1 de 2012 